See discussions, stats, and author profiles for this publication at: https://www.researchgate.net/publication/221170485

\title{
Maximal Lifetime Rate and Power Allocation for Sensor Networks with Data Distortion Constraints
}

Conference Paper · June 2007

DOI: 10.1109/ICC.2007.606 · Source: DBLP

CITATION

1

3 authors, including:

Subhrakanti Dey

University of Melbourne

161 PUBLICATIONS 1,971 CITATIONS

SEE PROFILE
READS

93

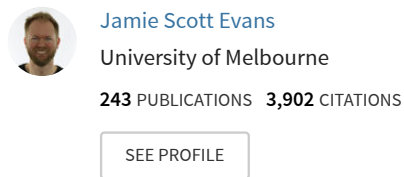




\title{
Maximal Lifetime Rate and Power Allocation for Sensor Networks with Data Distortion Constraints
}

\author{
James C. F. Li, Subhrakanti Dey and Jamie Evans \\ ARC Special Research Centre for Ultra-Broadband Information Networks (CUBIN) \\ Department of Electrical and Electronic Engineering \\ University of Melbourne, Victoria 3010, Australia \\ \{c.li, s.dey, j.evans@ee.unimelb.edu.au\}
}

\begin{abstract}
We address a lifetime maximization problem for a single-hop wireless sensor network where multiple sensors encode and communicate their measurements of a Gaussian random source to a Fusion Centre (FC). The FC is required to reconstruct the source within a prescribed distortion threshold. The lifetime optimization problem is formulated as a joint power, rate and timeslot (for TDMA) allocation problem under the constraints of the well known rate distortion constraints for the Gaussian CEO problem, the capacity constraints of the wireless links, the energy constraints of the sensor nodes and the strict delay constraint within which the encoded sensor data must arrive at the FC. We study the performances of TDMA and an interference limited non-orthogonal multiple access (NOMA) (with single user decoding) based protocols and compare them against the upper bound provided by the optimal lifetime performance where the capacity constraints are given by the Gaussian multiaccess capacity region. While the constrained non-linear optimization problems for the TDMA and the Gaussian multiaccess cases are convex, the NOMA case results in a non-linear nonconvex D.C. (difference of convex functions) programming problem. We provide a simple successive convex approximation based algorithm for the NOMA case that converges fast to a suboptimal lifetime performance that compares favourably against the upper bound provided by the Gaussian multiaccess case. Extensive numerical studies are presented for both static and slow fading wireless environments with full channel state information at the fusion centre.
\end{abstract}

\section{INTRODUCTION}

Wireless sensor networks (WSN) have become a key technology for the 21 st century due to its widespread applications in security, health, disaster response, defense, telecommunications, structural health monitoring etc. Due to limited energy resources and a distinct lack of centralized coordination (compared to cellular networks), the usefulness of these networks can become limited unless special care is taken to optimize energy consumption in communication and computation. Optimizing the lifetime of a WSN is thus an important problem. In many typical wireless sensor network applications, a set of nodes or agents measure or collect data from a source or phenomenon of interest (e.g, temperature in a bushfire prone area or surveillance pictures of human movements etc.) and then transmit them (possibly over a multi-hop relay network) to a sink or a base station where all data are collected and decisions or final estimates are made. In such a network, energy consumption is affected by such diverse parameters as choice of routes, MAC protocols, transmission scheduling, data rates, transmit power, wireless channel quality and fading etc. Thus, to optimize the lifetime of a WSN, one really has to consider a cross-layer design. This often leads to very complicated mixed-integer nonlinear optimization problems. Some such cross-layer issues with joint power and rate control

This work was supported by the Australian Research Council. CUBIN is an affiliated program of National ICT Australia (NICTA). have been studied in [1], [2]. In particular, [2] studied a joint power and rate control problem for lifetime optimization in a multi-hop wireless sensor network with constraints on outage induced by channel fading. In both [1], [2], nonconvex nonlinear optimization problems were transformed into approximate convex optimization problems and solved using sophisticated convex optimization tools. Lifetime optimization with joint rate and power control in interference limited ad hoc networks has also been considered in [3], whereas some earlier work has focused on specific key issues such as maximum lifetime routing algorithms such as [4], [5].

However, the nature of data being communicated via the WSN's considered in the above articles as well as in many other works (that cannot be mentioned here due to space limitations) were considered to be generic, and the only constraints (if any) on the rates of data transmission were dictated by flow conservation laws and the maximum link capacities. No particular attention was paid to the nature of the source of the data and the specific task performed by the WSN. Recently, however, a lifetime maximization problem was considered with rate distortion constraints in [6]. In this paper, the specific task for the WSN is considered to be reconstruction of a remote random source. It is well known from rate distortion theory that higher data rates may allow high quality data reconstruction (e.g, in surveillance camera applications), it may also result in large amount of energy expenditure in a WSN due to multi-hop transmission. This inherent trade-off between transmission rates (to achieve a certain pre-specified distortion threshold) and lifetime of the WSN was studied in [6] for an interference-free WSN where transmission power was kept fixed and the only optimization variables were the data rates in the various links of the WSN. The rate distortion constraints were given by the data rate constraints derived in [7] for sensors with unequal noise variances for the well known Gaussian CEO problem. In order to make their nonlinear optimization problem tractable, the authors of [6] made some judicious linear approximations and obtained upper and lower bounds on the optimal network lifetime using linear programming methods. It was shown in [8] however, that this nonlinear optimization problem can be transformed into a convex problem by a clever variable substitution and can be solved exactly by using standard convex optimization tools such as interior point methods.

Another key constraint in data communication over wireless sensor networks is the delay incurred in receiving the data, which may be critical in many applications such as video surveillance, disaster response scenarios, or networked control applications where actuators have to take timely decisions or actions to stabilize a remotely observed system via a WSN. In [9], the authors considered an energy optimal time scheduling 
problem with a strict delay constraint where a nonuniform time division multiple access scheme (TDMA) is used for downloading fixed amounts of data from various sensors into a fusion centre within a strict time duration. In our paper, we consider a similar single-hop sensor network where multiple sensors (agents) encode and transmit noisy measurements of a remote Gaussian source to a Fusion Centre (FC). The encoding rates for the various sensors can be different and are adjustable to suit the channel conditions and the delay constraint. The sensors or agents transmit their their encoded data at rates which may or may not be equal to the encoding rates, depending on the multiple access scheme and the delay constraint. Each sensor is equipped with a finite amount of initial energy. The wireless channel between each sensor and the fusion centre is taken to be static over the duration of the strict delay constraint. This delay duration is assumed to be long enough so that the maximum achievable data rate can be expressed as the Shannon capacity for that channel realization, but not too long so that the delay constraint loses its physical significance (see [9] for a similar assumption). The channels while being static during one time slot duration equal to the delay, can change randomly from one time slot to the next. In this paper we assume that the FC has perfect channel state information (CSI) within a specific delay duration time slot, since the channel fading is assumed to be slow.

We consider the problem of maximizing the lifetime of this sensor network with respect to transmit power and encoding rates (or transmission rates since they are related), subject to the data transmission rates satisfying the channel capacity constraints and the encoding rates satisfying the rate distortion constraints. The network lifetime is assumed to be long enough such that the sensor network is responsible for collecting measurements from the random source and transmitting them to the FC within the delay constraint a large number of times. This assumption essentially justifies the use of information theoretic capacity and rate distortion constraints in the formulation of the lifetime optimization problem. We consider both orthogonal time division multiple access (TDMA) (where an individual sensor transmits only for a fraction of the delay duration) and non-orthogonal interference limited multiple access (where all sensors transmit for the entire time slot but can create interference for each other) as the possible multiple access schemes. We also show that the ideal Gaussian multiaccess channel capacity constraints (assuming the sensor data are independent, although this is clearly not the case here) provide an upper bound on the achievable lifetime and use this as a benchmark to compare the lifetimes achieved by the TDMA and interference limited schemes. The corresponding optimization problems can be convex (Gaussian multiaccess and TDMA) or a nonconvex D.C. (difference of two convex functions) programming problem (interference limited case). We provide centralized solutions to the optimal power, rate and transmission duration (in the case of TDMA) allocation problems. The lifetime maximization problem for the Gaussian multiaccess case and the TDMA case can be solved globally using well know convex optimization tools. In order to solve the nonconvex optimization problem for the interference limited case, we use clever (similar to [10]) successive convex approximations of the original nonconvex optimization problem. In contrast to complex algorithms based on outer approximations or branch and bound methods [11] that take a long time to converge, we present practical algorithms that converge fast to suboptimal power and rate solutions for the interference limited case. These solutions, although suboptimal, compare favourably to the lifetime upper bound obtained by the Gaussian multiaccess case. Essentially, we argue that these algorithms can be run at the fusion centre and the optimal (or suboptimal) variables can be fed back to the sensors. Energy consumption is restricted to transmission only although this framework can be readily extended to include other forms of energy consumption such as due to sensing, reception and computation etc. Also, the fusion centre is assumed to have access to replenishable energy and therefore the energy consumption in feedback is not considered. Finally, we comment that we only focus on centralized (i.e, not distributed) optimization algorithms as the number of sensing agents we can consider in such networks is small in the context of our problem. This is mainly due to the fact that the number of rate distortion constraints increases exponentially with the number of sensing nodes.

\section{Problem Formulation}

The wireless sensor network considered in this paper is presented in Figure 1 where multiple sensor nodes or agents send their information to a fusion centre (FC) via wireless links. The set of sensor nodes is denoted by $\mathcal{V}$. The sensor nodes $\left\{A_{1}, A_{2}, \ldots, A_{|\mathcal{V}|}\right\}$ observe a Gaussian random source $X$ where $X \sim N\left(0, \sigma_{x}^{2}\right)$. The noisy measurement at the sensor $A_{i}$ is represented by $X+n_{i}$ where $n_{i} \sim N\left(0, \sigma_{i}^{2}\right)$. In general, the noise variances at the different sensor nodes are unequal, representing an inhomogeneous set of sensors. Agent $A_{i}$ encodes information at a rate $R_{i}$ and sends it to the fusion centre at a transmission rate of $R_{i}^{T}$. The transmission rate and the encoding rate may or may not be the same depending on the multiple access protocol. The FC has to reconstruct the source $X$ after receiving encoded measurements from all $|\mathcal{V}|$ sensors.

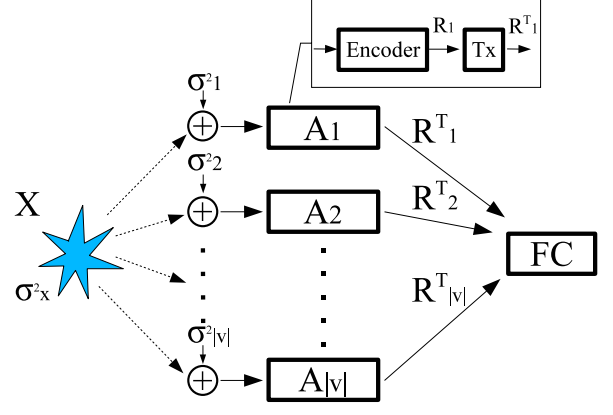

Fig. 1. Sensor Network measuring a random source

It is well known that the encoding rates from various sensors need to satisfy a set of rate distortion constraints to achieve a maximum distortion threshold. For the multi-sensor case, these results were obtained as the solution to the Gaussian CEO problem for the inhomogeneous sensor case in [7]. These constraints were rewritten in a slightly different form in [8] in the context of a lifetime optimization problem for a multihop sensor network. Below we quote these rate distortion 
constraints from [8], which can be easily shown to be convex:

$$
\begin{gathered}
\sum_{v \in \mathcal{W}_{k}} r_{v}+\frac{1}{2} \log \frac{1}{D_{t h}}-\frac{U_{k}}{2}-\sum_{v \in \mathcal{W}_{k}} R_{v} \leq 0 \\
k=1, \ldots, 2^{|\mathcal{V}|}-1 \\
e^{U_{k}}-\frac{1}{\sigma_{X}^{2}}-\sum_{v \in \mathcal{V} \backslash \mathcal{W}_{k}} \frac{1-e^{-2 r_{v}}}{\sigma_{v}^{2}} \leq 0 \quad k=1, \ldots, 2^{|\mathcal{V}|}-1 \\
\frac{1}{D_{t h}}-\frac{1}{\sigma_{X}^{2}}-\sum_{v \in \mathcal{V}} \frac{1-e^{-2 r_{v}}}{\sigma_{v}^{2}} \leq 0
\end{gathered}
$$

where $D_{t h}$ is the maximum allowed distortion threshold after reconstruction at the fusion centre and $\mathbf{r} \in \mathbb{R}^{+}$and $\mathbf{U} \in$ $\mathbb{R}$ are auxiliary variables. The set $\mathcal{W}$ contains all the nonempty subsets $\left\{\mathcal{W}_{1}, \mathcal{W}_{2}, \ldots, \mathcal{W}_{2^{|\mathcal{V}|}-1}\right\}$ of $\mathcal{V}$. Here, $\mathbf{R}=$ $\left[R_{1}, \ldots, R_{|\mathcal{V}|}\right]^{T}$ where $T$ denotes the transpose operation. Clearly, to achieve a distortion less than or equal to $D_{t h}$ at the fusion centre the amount of information FC fetches from the various agents needs to be great than or equal to $\mathbf{R}=\left[R_{1}, \ldots, R_{|\mathcal{V}|}\right]^{\prime}$ in bits/sec/Hz. The rate distortion region $\left(D_{t h}, \mathbf{R}\right)$ is called achievable when the inequalities (1) hold for the pair $D_{t h}$ and $\mathbf{R}$.

We assume that the sensors are sensing data at constant rates and the FC has to download all data encoded by each sensor within a strict time schedule $T$. This delay constraint essentially implies that all data encoded by sensor $A_{v}$ within a time duration $T$ have to be received at the $\mathrm{FC}$ within the same time duration for all $v$. Note that if the sensors are transmitting all the time, such as in an interference limited scheme, then this delay constraint is equivalent to having the transmission rate equal to the encoding rate. However, if a TDMA scheme is chosen for transmission, where $A_{v}$ transmits only for a duration $t_{v} T$, where $0 \leq t_{v} \leq 1$, and $\sum_{v} t_{v}=1$, then the transmission rate $\bar{R}_{v}^{T}=\frac{R_{v}}{t_{v}}$, which is clearly greater than the encoding rate. The channels between the sensor nodes and the FC are assumed to suffer distance based attenuation in the first instance and later assume that the channels additionally undergo independent identically distributed Rayleigh fading. In the case of fading, the channel dynamics are assumed to be slow enough so that the maximum achievable rate of transmission for each channel is given by the Shannon capacity for that particular channel realization, which is assumed to be static within the delay duration $T$ for all sensors. Consequently, we consider perfect CSI at the FC for the fading scenario as well.

Assuming that the $v$-th sensor is equipped with an initial energy $E_{v}$, we define the lifetime of the network as

$$
L T_{n e t} \triangleq \min _{v \in \mathcal{V}} L T_{v}=\min _{v \in \mathcal{V}} \frac{E_{v}}{P_{v}^{a v g}}
$$

where $P_{v}^{a v g}$ is the average power consumed in data transmission. This concept of lifetime is intuitive in the sense that it denotes the minimum time before the first node runs out of energy. Note that we do not consider any other form of energy expenditure such as sensing, computation etc. and also the energy expenditure at the FC is not taken into account since the FC is assumed to have access to a substantial energy reserve. In this paper, we seek to maximize this network lifetime (as given in (2) subject to the rate distortion constraints, the delay constraint and the channel capacity constraints mentioned above, by optimally allocating transmit power, encoding rates and (in the case of TDMA) transmission time duration at the various sensors. These optimization algorithms are performed as the FC and the optimal variables are fed back to the sensors with negligible communication delay. We consider the two usual multiple access schemes: an orthogonal TDMA and a non-orthogonal interference limited case and compare their performance with an upper bound provided by a (idealized) protocol that can achieve the Gaussian multiaccess capacity region for independent sensors. In the next section, we present the nonlinear optimization problems for the above three cases.
ADMA system

In a TDMA based transmission scheduling scheme, we allow the sensor node/agent $A_{v}$ to transmit during a fraction $t_{v}$ of the available time slot (which is also taken to be equal to the delay duration within which all data have to be downloaded into the fusion centre). Clearly, we have $\sum_{v \in \mathcal{V}} t_{v} \leq 1$. As alluded to earlier, the transmission rate of node $v$ is decided by the encoding rate $R_{v}$ in (1) and allocated timeslot $t_{v}$, and is given by $\frac{R_{v}}{t_{v}}$. In addition, the rate of transmission is upper bounded by the the continuous-time Shannon capacity (assuming full CSI at the FC) with channel bandwidth normalized to $(W=1)$ such that:

$$
W \log \left(1+\frac{g_{v} P_{v}}{N_{0} W}\right)=\log \left(1+\frac{g_{v} P_{v}}{N_{0}}\right) \geq \frac{R_{v}}{t_{v}}
$$

where $P_{v}$ is the transmission power for the $v$-th sensor node, $g_{v}$ denotes the propagation gain of the wireless channel between node $v$ and the FC, and $N_{0}$ is the average power of the background noise at the FC receiver.

Combining all the constraints (rate distortion, delay and capacity constraints) and the objective function (2), we have the following optimization problem for the TDMA scheme:

$$
\begin{gathered}
\max _{\mathbf{t}, \mathbf{P}, \mathbf{R}} L T_{n e t} \\
\text { s.t. } \quad \frac{E_{v}}{P_{v} t_{v}} \geq L T_{\text {net }} \quad v=1, \ldots,|\mathcal{V}| \\
\log \left(1+\frac{g_{v} P_{v}}{N_{0}}\right) \geq \frac{R_{v}}{t_{v}} \quad v=1, \ldots,|\mathcal{V}| \\
\sum_{v \in \mathcal{V}} t_{v} \leq 1
\end{gathered}
$$

$\left\{\mathbf{R}:\right.$ (1) holds for distortion threshold $\left.D_{t h}\right\}$

$$
\mathbf{t}, \mathbf{P}, \mathbf{R} \succeq 0 \text {. }
$$

It can be easily shown to be a non-linear nonconvex optimization problem. However, one can transform this nonconvex problem to a convex optimization problem by defining the following variables: $\bar{q}=\frac{1}{L T_{n e t}}, \bar{P}_{v}=t_{v} P_{v}$. One can then rewrite the energy and capacity constraints and transform the above nonconvex optimization problem into the following convex formulation, which can be solved by well established convex optimization tools based on interior point methods.

$$
\begin{gathered}
\min _{\bar{q}, \mathbf{t}, \overline{\mathbf{P}}, \mathbf{R}} \bar{q} \\
\text { s.t. } \quad-q E_{v}+\bar{P}_{v} \leq 0, \quad v=1, \ldots,|\mathcal{V}| \\
t_{v}\left(\exp \frac{R_{v}}{t_{v}}-1\right)-\frac{g_{v}}{N_{0}} \bar{P}_{v} \leq 0, \quad v=1, \ldots,|\mathcal{V}| \\
\sum_{v \in \mathcal{V}} t_{v} \leq 1
\end{gathered}
$$

$\left\{\mathbf{R}:\right.$ (1) holds for distortion threshold $\left.D_{t h}\right\}$

$$
\bar{q}, \mathbf{t}, \overline{\mathbf{P}}, \mathbf{R} \succeq 0 \text {. }
$$




\section{B. Interference Limited System}

In the interference limited scheme, all sensors transmit throughout the entire delay duration, and hence there always exists interference from other sensors which degrade the quality of reception at the FC. The capacity constraint on the transmission rate is then given by (note that here transmission rate is the same as the encoding rate and bandwidth has been normalized to $W=1$ )

$$
\log \left(1+\frac{g_{v} P_{v}}{\sum_{k \neq v} g_{k} P_{k}+N_{0}}\right) \geq R_{v}
$$

Note that in this formulation, each sensor sees the interference created by other sensors as noise and hence this scheme is non-orthogonal as opposed to the TDMA scheme. While this results in an inferior channel capacity, the FC uses a very simple single user decoding policy (instead of joint decoding), which allows for a low-complexity receiver. We call this simple multiple access scheme (6) as the non-orthogonal multiple access scheme (NOMA) in the rest of the paper.

Clearly, one can obtain better channel capacity and consequently, better network lifetime by considering multiple access schemes such as CDMA with multiuser detection such as linear minimum mean square error (LMMSE) receivers [12] or other more complex nonlinear receivers such as successive interference cancellation (SIC). Lifetime optimization problems for CDMA with complex multiuser detection schemes will be studied in a separate paper. We emphasize however, that the optimization technique developed in this paper for the interference limited case can be easily extended to cope with such problems.

Note that the energy requirements in the NOMA system are different to the ones in TDMA, since all sensor nodes are transmitting throughout the entire time the network is alive. Therefore, for all $v$, we have $\frac{E_{v}}{P_{v}} \geq L T_{\text {net }}$.

In summary, the lifetime maximization problem in the NOMA case is given by:

$$
\begin{gathered}
\max _{\mathbf{P}, \mathbf{R}} L T_{\text {net }} \\
\text { s.t. } \quad \frac{E_{v}}{P_{v}} \geq L T_{\text {net }} \quad v=1, \ldots,|\mathcal{V}| \\
\log \left(1+\frac{g_{v} P_{v}}{\sum_{k \neq v} g_{k} P_{k}+N_{0}}\right) \geq R_{v} \quad v=1, \ldots,|\mathcal{V}|
\end{gathered}
$$

$\left\{\mathbf{R}:\right.$ (1) holds for distortion threshold $\left.D_{t h}\right\}$

$$
\mathbf{P}, \mathbf{R} \succeq 0
$$

This optimization problem is non-linear and nonconvex. In Section III, we will propose a successive convex approximation based methodology to solve the optimization problem given by (7).

\section{Gaussian Multiaccess Channel}

It is obvious that in the problem we consider, the sensor nodes are transmitting to the FC via a Gaussian Multiaccess Channel. Note that here all sensors measure the same source, hence their data are correlated. Obtaining the capacity region for the Gaussian multiaccess channel with correlated sources is known to be a difficult problem. However, here we consider the capacity region for the Gaussian multiaccess channel (pretending that the sensor data are independent) and show that the corresponding optimal lifetime provides an upper bound on those achieved by the TDMA and NOMA schemes.
This capacity region is given by (with normalized bandwidth $W=1)$ [13]

$$
\begin{gathered}
\left\{\sum_{v \in \mathcal{W}_{k}} R_{v} \leq \log \left(1+\frac{\sum_{v \in \mathcal{W}_{k}} g_{v} P_{v}}{N_{0}}\right) ; \text { where } \mathcal{W}_{k} \in \mathcal{W}\right. \\
\left.k=1,2, \ldots, 2^{|\mathcal{V}|}-1\right\}
\end{gathered}
$$

Accordingly, the optimization problem can be described as

$$
\begin{gathered}
\max _{\mathbf{P}, \mathbf{R}} L T_{\text {net }} \\
\text { s.t. } \quad \frac{E_{v}}{P_{v}} \geq L T_{\text {net }} \quad v=1, \ldots,|\mathcal{V}| \\
\sum_{v \in \mathcal{W}_{k}} R_{v} \leq \log \left(1+\frac{\sum_{v \in \mathcal{W}_{k}} g_{v} P_{v}}{N_{0}}\right) \quad k=1, \ldots, 2^{|\mathcal{V}|}-1 \\
\left\{\mathbf{R}: \text { (1) holds for distortion threshold } D_{t h}\right\} \\
\mathbf{P}, \mathbf{R} \succeq 0 .
\end{gathered}
$$

The non-linear optimization problem (9) can be converted to a convex minimization problem simply after a single variable substitution $\bar{q}=\frac{1}{L T_{n e t}}$ :

$$
\begin{gathered}
\min _{\mathbf{P}, \mathbf{R}} \bar{q} \\
\text { s.t. } \quad P_{v}-E_{v} \bar{q} \leq 0 \quad v=1, \ldots,|\mathcal{V}| \\
e^{\sum_{v \in \mathcal{W}_{k}} R_{v}}-\frac{\sum_{v \in \mathcal{W}_{k}} g_{v} P_{v}}{N_{0}}-1 \leq 0 \quad k=1, \ldots, 2^{|\mathcal{V}|}-1
\end{gathered}
$$

$\left\{\mathbf{R}:\right.$ (1) holds for distortion threshold $\left.D_{t h}\right\}$

$$
\mathbf{P}, \mathbf{R}, \bar{q} \succeq 0
$$

Therefore, the rate and power allocation problems for the Gaussian multiaccess capacity region with rate distortion constraints can be solved using standard convex optimization tools. It can be shown in a fairly straightforward manner that the optimal lifetime solution of (10) provides an upper bound for the optimal solutions achieved by the TDMA and NOMA schemes, as the following result states. The proof can be found in the Appendix for the TDMA case, the proof for the NOMA case can be obtained in a similar fashion and is not provided due to lack of space. For a simple intuitive explanation of this result, see Chapter 6, pages 232-233 of [14].

Proposition 1: The optimal lifetime solution to the convex optimization problem for the Gaussian multiaccess rate region given by (10) provides an upper bound to the optimal lifetime solutions for nonconvex optimization problems for the TDMA and the NOMA cases given by (4) and (7), respectively.

III. SUCCESSIVE CONVEX APPROXIMATIONS FOR THE NONCONVEX NOMA PROBLEM

In Section II, we formulated the lifetime optimization problem with the rate distortion constraints for the NOMA environment which resulted in a non-linear nonconvex optimization problem. In fact, by suitable variable transformations, one can pose this nonconvex problem as a D.C. (representing difference of convex functions) programming problem which can be converted to a corresponding canonical D.C. programming problem. Canonical D.C. problems can be solved by outer approximation and branch and bound methods [11]. However, the complexity of D.C. programming is NP hard and convergence time is quite long [15]. In our work, we adopt a simpler strategy and approximate the original nonconvex problem (7) with a sequence of convex approximations. For a similar sequential convex optimization algorithm for power 
and rate allocation in an interference limited MANET, see [10]. Starting at some suitably chosen initial point, we solve an approximate convex problem and then use the results of this optimization procedure to obtain a new convex approximation of the original nonconvex problem. Thus this method leads to a sequence of convex problems which can be shown to converge (under certain conditions) to a sub-optimal solution. Below, we describe this successive convex approximation algorithm in detail for the NOMA case.

It was shown in [8] that the rate distortion constraints are convex in $\mathbf{R}, \mathbf{r}$, and $\mathbf{U}$. We now consider the energy and capacity constraints for the nonconvex NOMA problem (7).

Suppose we make the following variable transformations:

$$
L T_{n e t}=e^{q}, \quad t_{v}=e^{\tilde{t}_{v}}, \quad P_{v}=e^{\tilde{P}_{v}} \quad v=1, \ldots,|\mathcal{V}|
$$

Accordingly, the energy constraints in (7) can be represented in $q$, and $\tilde{P}_{v}$ as follows (after taking logarithm of both sides) $q+\tilde{P}_{v}-\log \left(E_{v}\right) \leq 0 \quad v=1, \ldots,|\mathcal{V}|$, which is obviously convex.

Now define an auxiliary variable $Q_{v}, v=1, \ldots,|\mathcal{V}|$ such that the capacity constraint can be rewritten as

$$
R_{v} \leq \log \left(1+e^{Q_{v}}\right) \quad \text { and } \quad e^{Q_{v}} \leq \frac{g_{v} e^{\tilde{P}_{v}}}{\sum_{k \neq v} g_{k} e^{\tilde{P}_{k}}+N_{0}} .
$$

Taking $\log$ on both sides of the inequalities, we can rewrite the above set of constraints as:

$$
\begin{gathered}
\log \left(R_{v}\right)-\log \left(\log \left(1+e^{Q_{v}}\right)\right) \leq 0 \\
\log \left(\frac{N_{0}}{g_{v}} e^{Q_{v}-\tilde{P}_{v}}+\sum_{k \neq v} \frac{g_{k}}{g_{v}} e^{Q_{v}+\tilde{P}_{k}-\tilde{P}_{v}}\right) \leq 0
\end{gathered}
$$

Here the second set of constraints are convex as they are in the standard log-sum-exponential form. It is the first set of constraints that are not convex. It is easy to demonstrate that $\log \left(\log \left(1+e^{Q_{v}}\right)\right)$ is a concave function in $Q_{v}$. However, note that $\log R_{v}$ is not convex, in fact, it is concave. Note that each of these nonconvex capacity constraints can be written as a difference of two convex functions and hence the original nonconvex problem can be converted to a canonical D.C. problem. Our strategy, however, is to approximate $\log R_{v}$ by a convex expression (as in [2]). Consider the tangent line that touches the concave curve $\log R_{v}$ at the point $\left(R_{0}, \log R_{0}\right)$. The equation representing this tangent line is obviously given by $y=\frac{x}{R_{0}}+\log R_{0}-1$ where $x$ refers to the $R_{v}$ axis. Consider another point $(R, \log R)$ nearby and the corresponding point on the tangent line with an ordinate value of $H_{R}=a_{v} R+b_{v}$, where $a_{v}=\frac{1}{R_{0}}$ and $b_{v}=\log R_{0}-1$. Clearly, $H_{R} \geq \log R$ since the tangent lies above the concave $\log R_{v}$ curve. It is now easily seen that if one satisfies the constraint

$$
\begin{gathered}
a_{v} R_{v}+b_{v}-\log \left(\log \left(1+e^{Q_{v}}\right)\right) \leq 0 \\
v=1, \ldots,|\mathcal{V}|
\end{gathered}
$$

with an appropriately chosen $\left(a_{v}, b_{v}\right)$, then the original NOMA capacity constraint is also satisfied. Thus, in general, a suboptimal solution to the original nonconvex lifetime maximization with rate distortion constraints in the NOMA case can be found by solving the following convex optimization problem

$$
\begin{gathered}
\min _{q, \tilde{\mathbf{P}}, \mathbf{R}, \mathbf{Q}}-q \\
q+\tilde{P}_{v}-\log \left(E_{v}\right) \leq 0 \quad v=1, \ldots,|\mathcal{V}| \\
a_{v} R_{v}+b_{v}-\log \left(\log \left(1+e^{Q_{v}}\right)\right) \leq 0 \quad v=1, \ldots,|\mathcal{V}| \\
\log \left(\frac{N_{0}}{g_{v}} e^{Q_{v}-\tilde{P}_{v}}+\sum_{k \neq v} \frac{g_{k}}{g_{v}} e^{Q_{v}+\tilde{P}_{k}-\tilde{P}_{v}}\right) \leq 0 \quad v=1, \ldots,|\mathcal{V}|
\end{gathered}
$$

$\left\{\mathbf{R}:\right.$ (1) holds for distortion threshold $\left.D_{t h}\right\}$

$$
\mathbf{R} \succeq 0 \text {. }
$$

A. A Successive convex approximation algorithm based on updating of tangent points

In the previous section, we illustrated how the nonconvex NOMA optimization problem can be converted to an approximate convex optimization problem by suitably choosing an initial point around which a tangent approximation is made, such that the resulting convex problem is feasible. Once this convex (approximate) problem is solved, the resulting rate $R_{v}$ can then be used as a new point of approximation to form a new approximate convex problem. Thus, one can form a sequence of convex approximations where the solution from the previous stage becomes the point of tangent approximation for the next stage. Below, we show that this sequence of convex approximations results in a sequence of optimal solutions that asymptotically converge to unique steady state values, provided that the initial convex approximate problem is feasible. In practice, one can stop this successive convex approximation method once a certain accuracy is reached in the optimal solution values.

Theorem 1: Suppose after solving the approximate convex problem (13) $k$ successive times, the logarithm of the inverse of the optimal lifetime value is given by $p^{(k)}$, i.e. $p^{(k)}=-q^{(k)}$, where $q^{(k)}$ is the logarithm of the achieved optimal lifetime value after solving $k$ successive convex approximations. If the initial convex approximation is feasible resulting in an optimal inverse lifetime $p^{(1)}$, then the sequence $\left\{p^{(k)}\right\}$ converges to a (not necessarily unique) steady state value $p^{*}$.

Proof: Suppose the feasible region for the $k$-th convex approximate optimization problem is $\mathcal{F}^{(k)}$. As above, the superscript $k$ means 'after $k$ updates'. We will show that sequence $\left\{p^{(k)}\right\}$ is nonincreasing and lower bounded, and consequently it converges.

\section{- Monotonicity:}

Suppose $\left[q^{(k)}, R^{(k)}, \tilde{P}^{(k)}, Q^{(k)}\right]$ is the optimal allocation scheme to the $k$-th convex approximation. Therefore, the parameters for the tangent approximation in the next round will be $a_{v}^{(k+1)}=\frac{1}{R_{v}^{(k)}}, b_{v}^{(k+1)}=\log R_{v}^{(k)}-1, v=$ $1, \ldots,|\mathcal{V}|$. We now show that $\left[q^{(k)}, R^{(k)}, \tilde{P}^{(k)}, Q^{(k)}\right]$ is within the feasible region $\mathcal{F}^{(k+1)}$ for the next iteration.

$$
\begin{aligned}
& a_{v}^{(k+1)} R_{v}^{(t)}+b_{v}^{(k+1)}-\log \left(\log \left(1+e^{Q_{v}^{(k)}}\right)\right) \\
= & \frac{1}{R_{v}^{(k)}} R_{v}^{(k)}+\log R_{v}^{(k)}-1-\log \left(\log \left(1+e^{Q_{v}^{(k)}}\right)\right) \\
= & \log R_{v}^{(k)}-\log \left(\log \left(1+e^{Q_{v}^{(k)}}\right)\right) \\
\leq & a_{v}^{(k)} R_{v}^{(k)}+b_{v}^{(k)}-\log \left(\log \left(1+e^{Q_{v}^{(k)}}\right)\right) \leq 0
\end{aligned}
$$

The last inequality follows from the fact that $\log R_{v}^{(k)} \leq$ $a_{v}^{(k)} R_{v}^{(k)}+b_{v}^{(k)}$ by virtue of the tangent approximation after the $(k-1)$ st iteration of the successive convex approximation scheme. Combining this with the 
fact that all the other constraints hold for the solution $\left[q^{(k)}, R^{(k)}, \tilde{P}^{(k)}, Q^{(k)}\right]$, we can conclude that

$$
\left[q^{(k)}, R^{(k)}, \tilde{P}^{(k)}, Q^{(k)}\right] \in \mathcal{F}^{(k+1)} .
$$

Since $p^{(k+1)}$ is the logarithm of the inverse optimal lifetime after $k+1$ updates, we have $p^{(k+1)} \leq p^{(k)}$.

- Boundedness: Note that once the network is activated, we can easily find $P_{v^{\prime}}$ for at least one activated sensor node $v^{\prime}$, where $P_{v^{\prime}}>0$. This implies that the lifetime of the network has a finite upper bound, since the initial node energy values are finite. It then follows that $p^{(k)}$ is lower bounded by a finite lower bound.

In summary, as a nonincreasing lower-bounded sequence (with a finite lower bound) $\left\{p^{(k)}\right\}$ converges (asymptotically) to a steady state value $p^{*}$ which implies that $q^{(k)}$ also converges to a steady state value and by uniqueness of the solution to each of the approximate convex problems, all other variables of optimization converge to their respective steady state values. Note that the choice of the first convex approximation may dictate the final steady state values, hence uniqueness of the steady state solutions cannot be guaranteed in general.

Although it is difficult to provide analytical results regarding the speed of convergence, we illustrate via simulations below that the successive convex approximation algorithm converges quite rapidly. These simulation results are for a six sensor network the details of which can be found in Section IV. Figure 2 illustrates the convergence for the NOMA case, where it is seen that after only 4 or 5 updates, the successive convex approximation based algorithm yields solutions within a reasonable accuracy. Recall that convergence is guaranteed as long as the problem is feasible at the initial iteration. These simulation results were achieved both by the MATLABbased fmincon program and well known Barrier Method based interior point techniques [16].

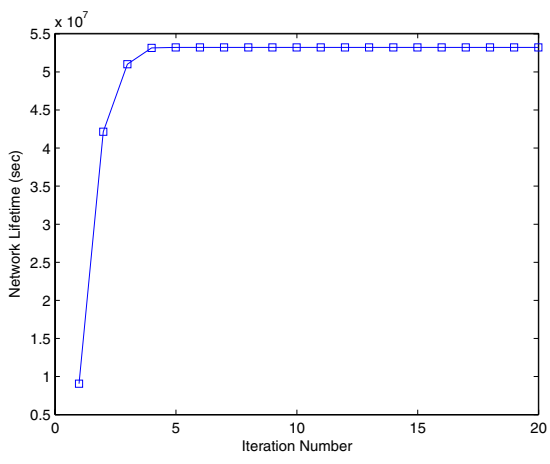

Fig. 2. Convergence of lifetime for the NOMA based WSN: $D_{t h}=-1 d B$

\section{Simulation STUdies}

In this section, we carry out extensive simulation studies mainly focusing on a 6-sensor single hop network where each sensor is connected via a wireless link to the fusion centre. The geometric configuration of this network is given in Figure 3, where the variance of the measurement is based on the distance between the source $X$ (representing the random phenomenon) and the sensor itself, $\sigma_{v}^{2}=\sigma_{0}^{2} d_{X v}^{2}$ [6]. The propagation gain for the wireless channel between the sensors and the fusion centre is proportional to the line-of-sight (LOS) distance with loss factor $n$. The distance parameters for the simulation

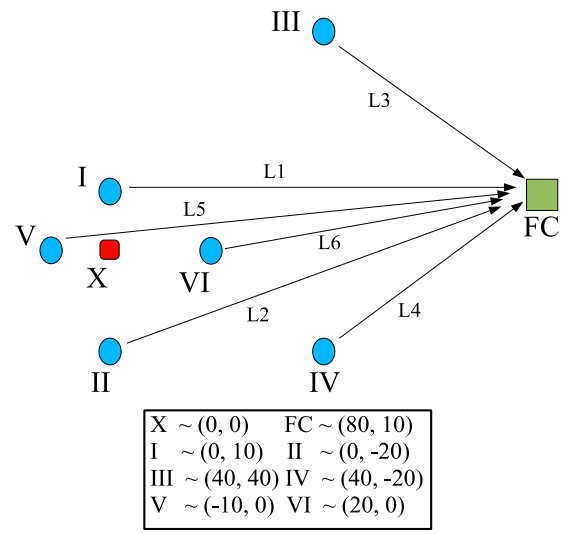

Fig. 3. Wireless Detection System with 6 Sensors (Unit: Meters)

studies with the 6-sensor network can be derived from Figure 3 , where the coordinates of the source, the sensors and the FC are given. All other relevant parameters are provided in Table I below.

\begin{tabular}{|l|c|}
\hline Frequency & $900(\mathrm{MHz})$ \\
\hline loss factor $n$ & 3 \\
\hline$\sigma_{0}^{2}$ & 0.01 \\
\hline$\sigma_{X}^{2}$ & 10 \\
\hline$N_{0}$ & $1 \times 10^{-12}($ Watts $)$ \\
\hline Bandwidth & $1(\mathrm{~Hz})$ \\
\hline$E_{v}, v=I, I I, I I I, I V, V, V I$ & $1000(\mathrm{Joules})$ \\
\hline
\end{tabular}

TABLE I

Simulation PARAMETERS

\section{A. Static Propagation Gain}

In this subsection, we assume that the link propagation gains between the sensors and the FC are deterministic and depend only on the distances between the sensors and the FC with a loss factor of $n=3$.

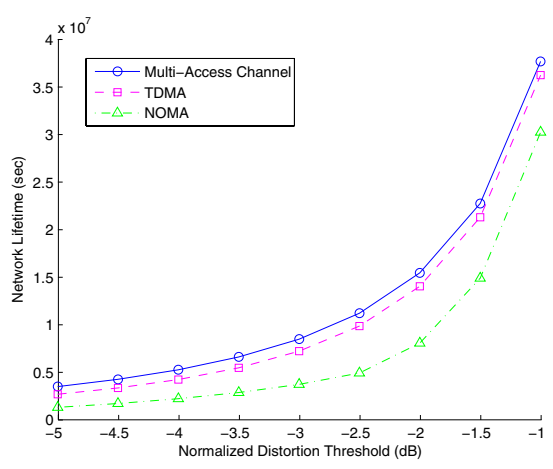

Fig. 4. Lifetime for the network with 4 Sensors

In Figure 4, we first illustrate the comparison among the results of three optimization problems (10), (5), and (13) for the 6-sensor situation. As expected, the network lifetimes for TDMA (optimal) and NOMA (suboptimal) are upperbounded by the solutions achieved by the constraints defined by the Gaussian Multiaccess Channel capacity region. The suboptimal solutions obtained by the sequential convex approximations for the NOMA case performs reasonably well, 
as compared to the upper bound provided the globally optimal solution to the Gaussian MAC case (albeit with the assumption of independence amongst sensor data). In these simulations, although TDMA seems to perform better, note that this may not always be the case depending on the network configuration and the relative distances amongst the nodes and the fusion centre.

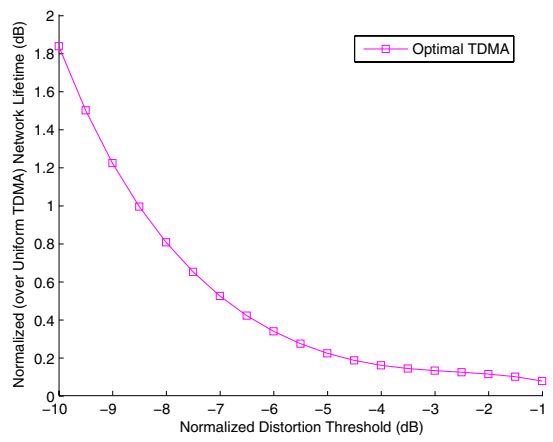

Fig. 5. Advantage of Optimal TDMA over Uniform TDMA

Figure 5 demonstrates the normalized improvement the network can achieve through an optimal TDMA scheme as opposed to a uniform TDMA scheme, where each sensor transmits for an equal $\frac{1}{6}$-th portion of the time. The $x$-axis is the normalized distortion threshold given by $10 \log \left(\frac{D_{t h}}{\sigma_{X}^{2}}\right)$. In the uniform TDMA case, the length of the activated time is the same for each sensor, hence the lifetime depends on the most power-consuming agent. On the other hand, optimal scheduling improves the performance by adaptively tuning the length of the individual sensor timeslots. Clearly, since uniform TDMA is a special case of a nonuniform TDMA, the optimal scheduling always performs better than uniform TDMA. However, when the distortion constraint is stricter, the improvement achieved by the optimal scheduling is more evident - since a lower distortion threshold requires higher transmission rate, the optimal assignment of timeslot fractions gives more flexibility to the network to improve its lifetime. performance of the network. Clearly, the normalized value of the optimal lifetime over that achieved by the uniform TDMA scheme decreases as the distortion threshold is relaxed.

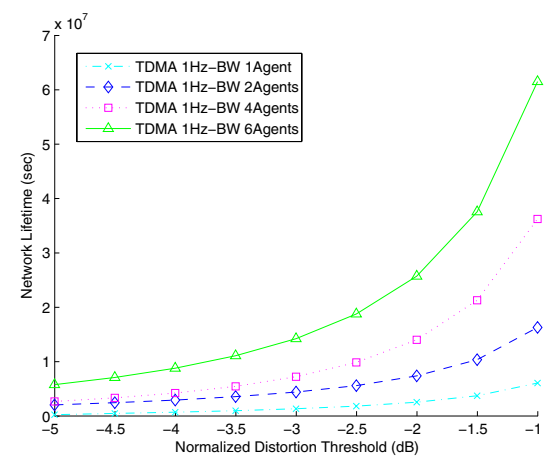

Fig. 6. Lifetime for the TDMA based Wireless Sensor Network

Figures 6 and 7 illustrate the relationship between the network lifetime and the number of sensor nodes (agents) in the system for the TDMA and the NOMA case respectively,

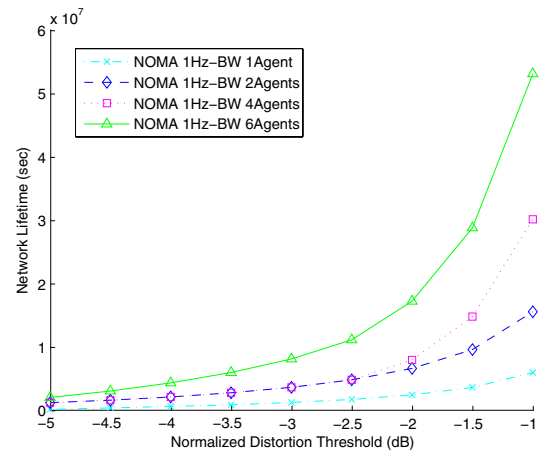

Fig. 7. Lifetime for the NOMA based Wireless Sensor Network

where the network consists of $1(\{\mathrm{II}\}), 2$ ( $\{\mathrm{I}, \mathrm{II}\}), 4(\{\mathrm{I}, \mathrm{II}$, III, IV $\})$, and 6(\{I, II, III, IV, V, VI $\})$ sensors along with the FC. In general, the network lifetime increases as the rate distortion requirement is relaxed. Also, in general, increasing the number of sensors in the TDMA case helps to extend the network lifetime due to the diversity available to the network. However, note that this is not always the case in the NOMA scheme, e.g, in the normalized distortion threshold domain of $(-5 \mathrm{~dB},-2.5 \mathrm{~dB})$ in the NOMA system, increasing the number of sensors from 2 to 4 does not give any advantage in lifetime performance, as shown in Figure 7. The reason is simple: nodes III and IV is too far from the phenomenon and hence it is not wise to use those two sensors when the distortion threshold is strict. Therefore, the optimal solution for the 4sensor case is to shut down node III and IV in order to eliminate the interference to the receiver. On the other hand, when the distortion threshold is less strict, the performance of the 4-sensor system will be better than that of the 2-sensor one.

\section{B. Slow Fading Environment}

In this subsection, we assume that the wireless link gains between the sensors and the fusion centre are not only path loss dependent, but also randomly varying due to slow Rayleigh fading. Under this assumption, the fading is slow enough so that even though the channel is random, it can be accurately estimated at the FC and and used to compute the optimal power, rate etc. which are fed back to the sensors. We assume that the propagation gain between the $v$-th node and the FC is effected by Rayleigh Fading parameters $F_{v}$, where $F_{v}$ has an exponential distribution with unity mean (without loss of generality), and $g_{v}$ (as before) represents the path loss dependent part. As a result, the channel capacity is given as

$$
\log \left(1+\frac{F_{v} g_{v} P_{v}}{N_{0}}\right) \quad \text { and } \log \left(1+\frac{F_{v} g_{v} P_{v}}{\sum_{k \neq v} F_{k} g_{k} P_{k}+N_{0}}\right)
$$

for the TDMA and NOMA systems respectively. Similarly, the capacity constraints for the Gaussian Multiaccess Channel case are given by

$$
\sum_{v \in \mathcal{W}_{k}} R_{v} \leq \log \left(1+\frac{\sum_{v \in \mathcal{W}_{k}} F_{v} g_{v} P_{v}}{N_{0}}\right),
$$

Figure 8, shows the optimal network lifetime obtained by the TDMA and NOMA schemes and the Gaussian MAC capacity limited case for the 4-sensor configuration studied 
before under slow Rayleigh fading (as described above) where the results are averaged over 1000 fading realizations. In order to make a fair comparison between the fading and no fading cases, the simulation results in Figure 4 (no fading) are repeated here. In Figure 8, all the lifetime curves in dotted lines represent the numerical results for the original non-fading cases, while those with solid curves represent the lifetimes achieved under a slow Rayleigh Fading environment. It is clear that the lifetime performance is comparable between the ones with and without such effects. Although fading shortens the network lifetime as a result of increasing transmission power to combat fading, the diversity available by using multiple sensors helps the network allocate resources to the sensors with better channel conditions and hence offset the power loss and extend the network lifetime.

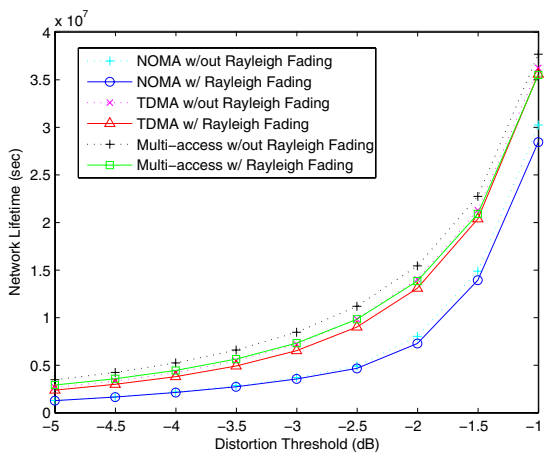

Fig. 8. Lifetime Performance in Rayleigh Fading - NOMA, TDMA, and Multiaccess Channel

\section{REFERENCES}

[1] R. Madan, S. Cui, S. Lall, and A. Goldsmith, "Cross-layer design for lifetime maximization in interference-limited wireless sensor networks," in IEEE INFOCOM'05, 2005.

[2] J. C. F. Li and S. Dey, "Lifetime optimization for wireless sensor networks with outage probability constraints," in Proc. European Wireless (EW 2006), April 2006.

[3] R. Jantti and S.-L. Kim, "Joint data rate and power allocation for lifetime maximization in interference limited ad hoc networks," IEEE Transactions on Wireless Communications, vol. 5, no. 5, pp. 1086-1094, May 2006.

[4] J.-H. Chang and L. Tassiulas, "Maximum lifetime routing in wireless sensor networks," IEEE/ACM Transactions on Networking, vol. 12, no. 4, pp. 1858-1863, August 2004.

[5] Y. Cui, Y. Xue, and K. Nahrstedt, "A utility-based distributed maximum lifetime routing algorithm for wireless networks," IEEE Transactions on Vehicular Technology, vol. 55, no. 3, pp. 797-805, May 2006.

[6] A. Kansal, A. Ramamoorthy, M. B. Srivastava, and G. J. Pottie, "On sensor network lifetime and data distortion," in IEEE ISIT '05, 2005, pp. 6-10.

[7] V. Prabhakaran, D. Tse, and K. Ramchandram, "Rate region of the quadratic gaussian ceo problem," in IEEE International Symposium on Information Theory, ISIT 2004, June 2004.

[8] J. C. F. Li and S. Dey, "Lifetime optimization for multi-hop wireless sensor networks with rate distortion constraints," in Proc. IEEE International Workshop on Signal Processing Advances for Wireless Communications (SPAWC) 2006, July 2006.

[9] Y. Yao and G. B. Giannakis, "Energy-efficient scheduling for wireless sensor networks," IEEE Transactions on Communications, vol. 53, no. 8, pp. 1333-1342, Aug. 2005.

[10] J. Papandriopoulos, S. Dey, , and J. Evans, "Distributed cross layer optimization of MANETs in composite fading," in Proc. IEEE International Conf. on Communications (ICC) 2006, July 2006.

[11] R. Horst and H. Tuy, Global Optimization: Deterministic Approaches. Springer-Verlag, 1993.

[12] J. Papandriopoulos, J. Evans, and S. Dey, "Optimal power control for Rayleigh-faded multiuser systems with outage constraints," IEEE Transactions on Wireless Communications, vol. 4, no. 6, pp. 2705-2715, Nov. 2005.

[13] T. Cover and J. Thomas, Elements of Information Theory. John Wiley and Sons, 1991.
[14] D. Tse and P. Viswanath, Fundamentals of Wireless Communication. Cambridge University Press, 2005.

[15] Y.-H. Lin and R. Cruz, "Power control and scheduling for interference links," in IEEE Information Theory Workshop 2004, October 2004, pp. 288-291.

[16] S. Boyd and L. Vandenberghe, Convex Optimization. Cambridge University Press, 2004

\section{APPENDIX}

Proof of Proposition 1:

Here, we provide the proof for the TDMA case only, the proof for the NOMA case can be derived in a similar fashion.

Consider the TDMA optimization problem given by (4) and the Gaussian MAC optimization problem given by (10) and note that it is the capacity constraints that set them apart.

Suppose the TDMA optimization problem has been optimized with respect to $t_{v}, v=1,2, \ldots,|\mathcal{V}|$ only and the resulting optimal solutions are given by $t_{v}^{*}, v=1,2, \ldots,|\mathcal{V}|$ Consider any nonempty subset $\mathcal{W}_{k}$ of $|\mathcal{V}|$. Note that feasibility of the solutions $t_{v}^{*}, v=1,2, \ldots,|\mathcal{V}|$ implies that $\sum_{v \in \mathcal{W}_{k}} t_{v}^{*} \leq$ 1 (in fact it can be shown that $\sum_{v \in \mathcal{V}} t_{v}^{*}=1$ ). This implies that summing over the TDMA capacity constraints: $R_{v} \leq$ $t_{v}^{*} \log \left(1+\frac{g_{v} P_{v}}{N_{0}}\right)$ for $v \in \mathcal{W}_{k}$, we get

$$
\begin{aligned}
\sum_{v \in \mathcal{W}_{k}} R_{v} & \leq \sum_{v \in \mathcal{W}_{k}} t_{v}^{*} \log \left(1+\frac{g_{v} P_{v}}{N_{0}}\right) \\
& \leq \log \left(1+\sum_{v \in \mathcal{W}_{k}} \frac{t_{v}^{*} g_{v} P_{v}}{N_{0}}\right) \\
& \leq \log \left(1+\sum_{v \in \mathcal{W}_{k}} \frac{g_{v} \bar{P}_{v}}{N_{0}}\right), \forall k=1, \ldots, 2^{\mid \mathcal{V}}(14)
\end{aligned}
$$

where $\bar{P}_{v}=t_{v}^{*} P_{v}$ and the second last inequality follows as a special case of Jensen's inequality.

Using the transformation $\bar{P}_{v}=t_{v}^{*} P_{v}$ one can now rewrite the energy constraints in the TDMA problem as $\frac{E_{v}}{\bar{P}_{v}} \geq L T_{\text {net }}, \quad \forall v \in \mathcal{V}$, where $\bar{P}_{v} \geq 0$. Denote $\overline{\mathbf{P}}=$ $\left(\bar{P}_{1}, \bar{P}_{2}, \ldots, \bar{P}_{|\mathcal{V}|}\right)$.

Therefore the new transformed TDMA problem (after optimizing over $t_{v}$ ) can be written as

$$
\begin{aligned}
& \max _{\overline{\mathbf{P}}, \mathbf{R}} L T_{n e t} \\
& \text { s.t. } \quad \frac{E_{v}}{\bar{P}_{v}} \geq L T_{n e t} \quad v=1, \ldots,|\mathcal{V}| \\
& t_{v}^{*} \log \left(1+\frac{g_{v} \frac{\bar{P}_{v}}{t_{v}^{*}}}{N_{0}}\right) \geq R_{v} \quad v=1, \ldots,|\mathcal{V}| \\
& \left\{\mathbf{R}:(1) \text { holds for distortion threshold } D_{t h}\right\} \\
& \overline{\mathbf{P}}, \mathbf{R} \succeq 0
\end{aligned}
$$

Clearly, the capacity constraints in the above problem imply (due to inequality (14)) the capacity constraints in the Gaussian MAC problem. Hence an optimal solution of the TDMA problem (4) belongs to the feasible set of the Gaussian MAC problem (10). Therefore, the optimal lifetime obtained from the Gaussian MAC problem is equal or greater than that obtained by an optimal solution of the TDMA problem. 